\title{
Security Utilization Of Cloud Computing In The World Of Business For Small Medium Enterprises (SMES)
}

\author{
Fachmi Tamzil $^{1}$, Nizirwan Anwar ${ }^{2 *}$, Muhammad Abdullah Hadi ${ }^{3}$ \\ ${ }^{1}$ Faculty of Economics and Business, Esa Unggul University, Jakarta 11510 \\ ${ }^{2,3}$ Faculty of Computer Science, Esa Unggul University, Jakarta 11510 \\ ${ }^{*}$ Corresponding Author: \\ Email: nizirwan.anwar@esaunggul.ac.id
}

\begin{abstract}
.
At this time the business world began to compete globally with the aim of satisfying customers, so companies need access and information that is fast and accurate. To access information quickly, companies need to build information and communication technology infrastructure which is certainly not easy with the need for a large enough capital, besides that there is cloud computing which can be an alternative. The progress of information and communication technology that we have achieved now has really been recognized and felt to provide a lot of convenience and comfort for human life. Information Technology (IT) solutions, cloud computing, are now starting to be discussed a lot by business people, including in Indonesia. Even though it's popular, it doesn't mean cloud computing is easily accepted. Cloud computing will actually save business operations much more, compared to using traditional methods among Small and Medium Enterprises (SMEs). There are various kinds of services provided by cloud computing, including hardware, infrastructure, platforms, and applications. Cloud computing has considerable benefits, this is because the services of cloud computing can reduce computing costs, increase reliability and provide considerable opportunities for the infrastructure world. The purpose of this research is to increase business competitiveness in the business sector by using the Salesfoorce App Cloud application. The results of this application can make business models more flexible and know market needs and consumer needs.
\end{abstract}

Keywords: Cloud, Small and Medium Enterprises (SMEs),

\section{INTRODUCTION}

Cloud computing is one example of the development of information technology. Cloud computing is the transformation of information and communication technology from client or server-based computers [1]. Cloud computing allows users to use software services, storage media, infrastructure platforms and technology service applications over the internet. Cloud computing technology benefits users because they no longer need to invest. Be a priority in almost all areas of business[2][3]. Today's large-scale or small-scale businesses are very developed with data as the center. They usually spend large sums of money to maintain that valuable data.That's why strong IT support with high-performance centralized storage is so important. Not all businesses can afford the high costs of in-house IT infrastructure. So for them, Cloud Computing is massive for software and data applications[4] as well as hardware maintenance. 
Cloud Computing is the process of processing computing [5][2][4] power (both CPU, RAM, Network Speeds, Software, OS and Storage) over a network (usually via the internet). So the data transfer that occurs is not physical and the computing resources owned are at the location of the users who use the service.With the increasing number of computer users and mobile users, this data storage is a good solution[6][7] because it is cheaper. There may also be other considerations of the company such as efficiency in storing data, calculations, and less maintenance costs.Cloud computing actually reduces the demand for hardware and software from the user's side. The only thing that the user must be able to run/do is the software interface of the cloud computing system, which can be as simple as a web browser. This can certainly help reduce user work with Cloud network technology that is responsive and automatically solves other IT problems.

When accessing/using an email service, the data will be stored on the cloud server, not on the computer. The technology and infrastructure[1] behind the cloud is invisible[8][9]. So, it doesn't matter whether the cloud service is based on HTTP, XML, Ruby, PHP or any other specific technology as long as it's still user-friendly and functional of course. As a user, you can connect to the cloud system from personal devices such as laptops or cellphones.Cloud computing also does make effective use of small businesses with limited resources. This technology or service can give small and medium businesses[10] access to technology that was previously out of their reach. Cloud computing is now helping a lot of small businesses to turn their expenses into profits. The main advantage of this technology is the potential for cost savings. Therefore, there are a lot of good companies from small to large scale businesses using this cloud service. Cloud computing is also able to provide freedom for users to be able to use services according to their respective needs and pay according to existing usage[11][12], so it is more flexible. Without spending a lot of internal resources, you can run IT operations well.

To find out the advantages of this cloud technology, please read the following points:

- Lowering the cost of IT infrastructure (computers, etc.) for its users

- Improve work performance or performance

- Reduces the risk of frequent and expensive maintenance because there are fewer problems

- Get instant updates to existing software Improve compatibility between operating systems used

- Provides backup/backup and recovery

- Improve scalability

- Increase storage capacity

- Improve data security 
There are four different types of cloud [4] that can be used, according to business needs. Here are the business requirements

- Private Cloud

This private cloud means that the cloud resources are used for one particular organization only (privately, not shared with other users/organizations). This method is mostly used for interactions such as intra-business, where the cloud resources can be managed, owned, and operated by the same organization.

- Community Cloud

Community cloud refers to the use of resources for communities and organizations.

- Hybrid Cloud

This type of cloud can be used for both types of interactions - B2B (Business to Business) or B2C (Business to Consumer). So, computing resources are tied together but with different clouds.

- Cloud Computing

The term cloud computing comes from various perspectives in academia and practice, which explains the variation in definitions. Buyya et al. (2009) described cloud computing as a 'fifth utility' along with water, gas, electricity and telephones. Based on this description, cloud computing includes available and on-demand access to computing services such as other utilities. NIST provides a definition of cloud computing by describing it as a model for enabling anywhere, convenient, on-demand network access to a configurable and shareable pool of computing resources. This study will use this definition because it describes the cloud model using five important characteristics, three models services, and four deployment models.

\section{METHODS}

The method used for data collection in this research is a literature study, where data collection is done by searching and studying data from books, journals or other references related to this research and observing SMEs. namely journals that have been seminars. This method approach is expected to provide and broaden insight to readers and specifically to SMEs related to the use of cloud computing in carrying out business processes. The method used for data collection in this research is a literature study, where data collection is done by searching and studying data from books, journals or other references related to this research and observing SMEs. namely journals that have been seminars. This method approach is expected to provide and broaden insight to readers and specifically to SMEs related to the use of cloud computing in carrying out business processes.

According to in the e-book "Cloud computing strategies" by Dimitris N. Chorafas explained that Cloud Computing as an On-Demand Technology [13][14], namely Cloud Computing technology is a technology based on requests from users. This technology is one point of change (Inflection Point), not only software 
applications based on Cloud Computing also includes platforms, database infrastructure and services that can be based on Cloud Computing [18].

Based on the recommendations of NIST (National Institute of Standards and Technology), there are 5 main characteristics in cloud computing[15][16]:

1) On-demand self-service: companies can order and determine the computing capabilities they want, and can be fulfilled quickly. For example: procuring a server in a company that takes weeks can be fulfilled in just minutes by a cloud provider.

2) Broad network access: companies can access this service widely from various types of devices such as smart phones, tablets, laptops, and PCs (personal computers).

3) Resource pooling: service providers have very large computing resources that can be dynamically allocated to serve many companies (multi-tenants) according to company requests.

4) Rapid elasticity: the ability of computing resources that can be changed quickly at any time according to company needs. For example: an online transportation application provider company such as Grab, which was initially only used by a few users, can increase the capacity of this service quickly and easily when its user growth reaches 200 times.

5) Measured service: the ability to control and measure resource utilization according to its use (usually on a pay-per-use or charge-per-use basis). For example: size of data stored, length of use, number of users.

\section{RESULT AND DISCUSSION}

The results of this study will be presented as follows:

\section{a. Cloud Computing Adoption Stages}

The stages in adopting cloud computing are as follows:

1). Analysis Stage

This initial stage, the user must carry out a SWOT analysis, to understand the user's needs in order to determine whether the project is feasible namely, feasibility, legal, compliance, organizational change governance and risk management.

\section{2). Planning Stage}

Is the stage of selecting a cloud service platform, application and infrastructure that is suitable for the needs of the organization to determine the costs that must be incurred by the organization. In preparing an adoption plan it is very important to decide whether cloud services will be officially used. Before it is officially used, will there be

pilot project to identify the risks that occur.

\section{3). Adoption Stage}

This stage is the preparatory stage for migration from conventional systems to actual cloud service infrastructure and applications. In the system/application phase this integration is carried out to ensure that candidate applications will be able to function with internal applications that are not migrating to the cloud and also with the 
cloud infrastructure. Outsourcing strategies are decided and benchmarks developed at the planning stage are used to measure the vendor's ability to provide services that will not affect the organization's delivery of services and businesses. The last thing in this phase is the development and signing contract that fulfills the user's need to deploy the cloud service.

\section{4). Migration Stage}

This stage concludes to prepare for migration to the cloud and can continue the migration. Application and data migration can be continued.Support for users during the migration process is provided, monitoring and control of the project is maintained to ensure a successful migration.

\section{5). Management Stage}

The project should now be fully operational in the cloud, but contract and vendor management, testing and maintenance, user support and reviews should be ongoing for the next few months to start. System measures that are developed as indicators of project success and must be monitored such as security, SLA, legal, and cost management are desirable metrics. Documentation of lessons learned and best practices during the project must be documented and communicated to all stakeholders.

\section{b. Reasons for Using Cloud Computing.}

There are 9 reasons to use cloud computing, namely:

\section{1). Reduce Cost}

Users of cloud technology, do not need to build cloud infrastructure, whether software, hardware, servers, networks or bandwidth, because all are provided by cloud service providers. Users only need to pay rent according to their operational needs. Thus, the user will save costs.

\section{2). increase}

Storage Capacity Cloud users can determine the amount of storage capacity according to their needs, eliminating the need to upgrade computer memory.

\section{3). Automation}

With cloud computing technology, users do not need to form a team to handle system updates and back-ups. Organizations no longer need to hire people to upgrade one or more servers. The server will always be updated by the vendor or cloud service provider according to user or company requests.

\section{4). Flexible}

Cloud computing offers more flexibility than conventional computing methods, has more flexibility than other network computing systems and saves time and money for busy and resource-less users.

5). More Mobility

Users can access information wherever they are/ubiquito for 24 hours, meaning that work can be done anywhere using smartphones, tablets, laptops as long as they have a connection and access to the internet. 


\section{6). IT Enabling}

To Stay Focused No longer having to worry about regular server updates and other computing issues, companies/users can be free to concentrate on company product innovation without thinking about how the infrastructure is running.

\section{7). Sharing Resources}

Cloud computing technology has the ability to share resources (memory, storage, bandwidth, and others) within the company. This allows all employees to access resources through cloud computing. This again saves time and money by placing resources in one location that is easy for employees to find and access.

8). Business Agility One of the advantages of cloud computing is that it allows companies to be more agile.

The speed of ordering capacity and the services provided by the vendor are important elements of cloud computing. The addition of storage media capacity, network bandwidth, memory, and other computing resources can be done quickly. Most cloud computing vendors use a software infrastructure that can easily add, move, or modify applications according to user requirements. It is the dynamic and elastic nature of cloud computing that will give it a big advantage.

9). One Back-up and Disaster Recovery Package With cloud computing, the burden of managing the technology is placed on the vendor including all things related to data protection, fault tolerance, recovery and disaster recovery.

With the cloud-based model, disaster recovery costs are estimated to be less than a one-time cost, a significant savings.

The integrity of cloud computing in SMEs depends on factors such as the organization, the technological context, and the environment. With the technology, it is hoped that it can have a positive impact on business people, especially SMEs. However, SMEs may delay the use of new technologies due to inadequate security considerations.

1). Cloud Computing Security Threats and Risks in SMEs. Broadly speaking, the security problems faced by SMEs in the cloud environment are contained in five categories including network problems, security standards, cloud infrastructure, access control, and data issues. Network-related issues include threats posed by DoS and DDoS attacks, man in the middle (MITM) attacks, flooding attacks, DNS attacks, and IP vulnerabilities. There are also concerns about dependence on the Internet and network security configurations. Security issues include the lack of adequate security standards to ensure strong security for the company, trust issues when working over the Internet, and compliance issues.

The existing literature focuses more on the available standards needed to mitigate cyberattacks on cloud infrastructure and the policies that can protect against cloud systems. For cloud infrastructure, key security concerns for SMBs include insecure API interfaces, security misconfigurations, server locations and data backups, and the multi-tenancy characteristics of cloud environments. The access control theme 
alludes to various security issues such as malicious insiders, authentication mechanisms, account and service hijacking, and privileged user access. This impact is unique to each business. Lastly, SMBs also have common security concerns regarding data protection, integrity, availability, and risk of data loss and privacy concerns so a multi-level solution is required.

2). Mitigation Measures for Cloud Computing Security Threats in SMEs. To address the second research objective, this study examines current cyber security practice standards, and measures, threat mitigation techniques for security issues in the context of cloud computing, and approaches to cloud security risk management. From the outset, research findings indicate that the most common security methods used by enterprise firewalls, physical network segregation, role-based access control mechanisms, data encryption, and identity management, and backup media monitoring. This study found that SMEs use security tools such as firewall technology, security intelligence systems, access governance tools, perimeter controls, and tools for automated policy management. While there is no surefire path to success in implementing security measures, the findings demonstrate maturity in terms of security practices and standards designed to guide enterprises.

Examples of best practices and guidance include the CyberNIST Security Framework, the ISO/IEC 27000 standard, and Critical Security Controls (CIS). To reduce security threats to the cloud, this research establishes the need for SMEs to focus on risk management. This study determined that SMEs[12][2][17] are increasingly realizing the importance of risk management, but that effective risk management remains a challenge for many businesses. The problem is that traditional approaches to risk management tend to focus on adopting a common methodology that addresses risk for all types of organizations. Given the unique nature of SMEs in terms of their security posture, this methodology would be too complex and unsuitable. However, further analysis has identified the CloudWatch2 methodology as a suitable tool for SMBs. CloudWatch is justified because it provides a simplified and easy approach to risk assessment in the cloud. For SMBs, CloudWatch's compatibility makes it possible to assess the SME's security posture, select appropriate security controls, and deploy and monitor risk profiles.

3). Implementation of the Cloud Computing Security Model for SMEs. Strategies to implement a good and secure cloud for SMEs. This strategy is modeled using the SABSA[18] Security architecture. The proposed conceptual model integrates four components: Cloud Model[10][19], Security Model[20], Compliance Model[18], and Security Major. The Cloud Model is the first step in an overall security strategy for SMEs, and it answers the questions "What" should be protected and "Who" will be involved in enterprise security management. For SMEs seeking to use cloud computing technology, the first step in the cloud deployment strategy considered in this model is to identify the type of system to be deployed and determine who will use the system. This approach enables SMEs to map out security architectures and define business, 
regulatory, and compliance requirements. This model identifies various sources of threats in cloud computing, including cloud actors, cloud deployment models, and cloud service models.

\section{CONCLUSION}

Cloud Computing technology has benefits and good goals for the development of technology that we use today, especially its use in business and other things such as in banking, finance, internet of things and other things that can facilitate a lot of human work. Cloud Computing technology itself will continue to develop along with the times and is also supported by adequate infrastructure throughout the region, especially in Indonesia. The hope is that cloud computing can be a solution and provide many benefits that can be felt by modern society today.

Cloud computing in its development is a technology that has fast and efficient capabilities, and can minimize data. In its implementation, cloud computing requires hosting that has a high and fast level of security, one of which is IDCloudhost which is the Number 1 hosting service provider in Indonesia, which has collaborated with companies and governments in managing hosting. Based on the overall discussion and results regarding the use of cloud computing, it is highly recommended for existing SMEs to use cloud computing services in running a business. Future developments are expected to emphasize the risks and security of cloud computing technology when used by SMEs.

\section{REFERENCES}

[1] S. Srivastava, S. Agarwal, R. Dubey, A. Murarka, and ..., "Scope of Cloud Computing in Business: A Compendious and Methodical Analysis of Trends in Publications and Patents," Vision, 2021.

[2] A. Kaur et al., "Security Recommendations for Cloud Computing Providers," Cloud Secur. Alliance, 2013.

[3] D. Lammie, "Cloud Computing in the Business World," Available SSRN 3830144, 2021.

[4] M. H. Mohamed Basel Almourad, "The Impact of Cloud Computing on the Channel," vol. 19, no. September, p. 10, Sep. 2011.

[5] M. Y. Sokiyna, M. J. Aqel, and O. A. Naqshbandi, "Cloud computing technology algorithms capabilities in managing and processing big data in business organizations: Mapreduce, hadoop, parallel programming," J. Inf. ..., 2020.

[6] C. I. Fan and S. Y. Huang, "Controllable privacy preserving search based on symmetric predicate encryption in cloud storage," Futur. Gener. Comput. Syst., 2013.

[7] Z. H. U. Liehuang, D. Hui, and S. Meng, "Privacy protection mechanism for blockchain transaction data," Big Data Res., 2018.

[8] T. Acar, M. Belenkiy, and A. Küpçü, "Single password authentication," Comput. Networks, 2013.

[9] D. W. Chadwick and K. Fatema, "A privacy preserving authorisation system for the cloud,” J. Comput. Syst. Sci., 2012. 
[10] A. Kaur and A. Verma, "An Abstract Model for Adaptive Access Control in Cloud Computing," Proc. Int. Conf. Recent ..., 2018.

[11] M. Ula, "Analisis Metode Pengamanan Data pada Layanan Cloud Computing," TECHSI-Jurnal Tek. Inform., 2019.

[12] E. Mohabbattalab, T. von der Heidt, and ..., "The perceived advantages of cloud computing for SMEs," GSTF Journal on .... Citeseer, 2014.

[13] D. N. Chorafas, Cloud computing strategies. books.google.com, 2010.

[14] B. J. S. Chee and C. F. Jr, Cloud computing: technologies and strategies of the ubiquitous data center. books.google.com, 2010.

[15] W. Jansen and T. Grance, "Guidelines on Security and Privacy in Public Cloud Computing (No. NIST SP 800-144). NIST-national Institute of Standards and Technology." 2011.

[16] W. Jansen and T. Grance, "Guidelines on security and privacy in public cloud computing. NIST: National Institute of Standards and Technology.” ... Rep. 800-144, 2011. Accessible: http ..., 2013.

[17] W. Long, S. Li, and X. Song, "Environmental regulation and the environmental performance of small and medium-sized enterprises (SMEs): Evidence from the SME board and growth enterprise ...," J. Public Adm, 2015.

[18] A. M. Mustapha, O. T. Arogundade, and ..., "A model-based business process compliance management architecture for SMSE towards effective adoption of cloud computing," ... Comput. ..., 2017.

[19] K. Riad and Z. Yan, "EAR-ABAC: An extended AR-ABAC access control model for SDN-Integrated cloud computing," International Journal of Computer Applications. researchgate.net, 2015.

[20] Y. A. Younis, K. Kifayat, and M. Merabti, "An access control model for cloud computing,” J. Inf. Secur. ..., 2014. 